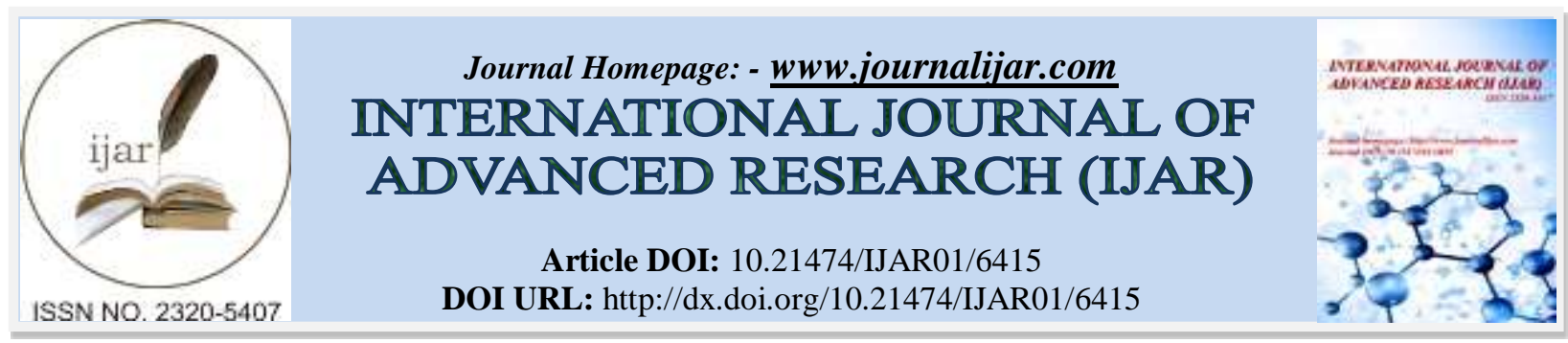

RESEARCH ARTICLE

\title{
PREVALENCE OF ATHEROSCLEROTIC CRDIOVASCULAR DISEASE MODIFIABLE MAJOR RISK FACTORS: A REVIEW.
}

\author{
Mohammad Ahmad Alamoudi ${ }^{1}$, Sultan Affan Bin Mahfooz ${ }^{1}$, Amar Abdullateef Softah ${ }^{1}$, Abdulrhman \\ Abdullateef Abdullah Softah ${ }^{2}$, Faris Jamal Merdad ${ }^{1}$, Abdulraheem Salem Almalki ${ }^{1}$, Abdullah Din \\ Mohammed $^{1}$, Rayan Mohammed Salih Al-Juaid ${ }^{1}$, Khalid Mohammed Aljuaid ${ }^{1}$, Yasmeen Marwan \\ Ghandoora ${ }^{1}$, Haya Amer Alabbassi ${ }^{1}$, Khaled Fahad Alsolami ${ }^{2}$ and Raed Talat Miralam ${ }^{1}$. \\ 1. Batterjee Medical College. \\ 2. Taif University, College of Medicine.
}

\section{Manuscript Info}

Manuscript History

Received: 02 December 2017

Final Accepted: 04 January 2018

Published: February 2018

Key words:-

Prevalence, Diabetes, Hypertension,

Obesity or over weight,

Dyslipidemia, Smoking, Physical

Inactivity, Saudi Arabia.

\section{Abstract}

Introduction: Globally, Atherosclerotic Cardiovascular disease is responsible for 17.7 million deaths yearly, representing almost one third of deaths globally. Its modifiable risk factors are hypertension, diabetes, overweight or obesity in addition to smoking, and physical inactivity. Saudi economic evolution over the past decades brought up significant increase in life expectancy and substantial modifications in lifestyle as well as it promoted prevalence of such risk factors.

Objective: to shed light on the prevalence of ASCVD major modifiable risk factors and their trend if any in Saudi Arabia.

Methodology: We searched published literatures that have addressed prevalence of modifiable risk factors of atherosclerotic cardiovascular disease in Saudi Arabia via the internet, using the medical database MEDLINE/PubMed. Retrieved articles were reviewed by authors. We excluded hospital-based studies or none original papers. Selected articles that matched the criteria were included in this review.

Results: in this review article we reviewed 21 epidemiological articles that highlighted the prevalence of atherosclerotic cardiovascular disease modifiable risk factors in Saudi Arabia. The real challenges are some risk factors are asymptomatic and underdiagnosed, poorly controlled and they are linked in a way that addition of one risk factor may lead to another one or aggravate it. The trend of such risk factors is growing, and programs should be in place to slow down their growth.

Conclusion: There is a need for more comprehensive awareness programs to publics aiming to promote healthy lifestyle and enhance early detection and adherence to treatment.

Copy Right, IJAR, 2018. All rights reserved.

\section{Introduction:-}

Atherosclerotic cardiovascular disease (ASCVD) entails variety of presentations depending on site of atheroma. Coronary atherosclerosis may present in the form of acute coronary syndromes (ACSs), Ischemic heart disease 
whether stable or unstable angina. Cerebrovascular atherosclerosis may present as transient ischemic attack, or stroke. Peripheral arterial disease may result from significant atheroma in peripheral arteries. [1]

ASCVD is directly accountable for 17.7 million deaths yearly, representing almost one third of deaths globally. Risk factors for ASCVD, which manifest initially as heart attacks and strokes, are hypertension, diabetes, overweight or obesity in addition to smoking, unhealthy diet, physical inactivity and the harmful use of alcohol. These risk factors are luckily either modifiable or controllable which make them a key to downgrade the risk of ASCVD. [2]

The challenge is that the prevalence of such risk factors in developing countries is increasing because of economic evolution, urbanisation, industrialisation and globalization. Also, sharp increase in life span prolongs the exposure to such risk factors that promote heart disease. [3]

Saudi Arabia witnessed economic evolution over the past decades that brought up significant increase in life expectancy and substantial modifications in lifestyle as well. This review aims to shed light on the prevalence of major modifiable risk factors for ASCVD and their trend if any in Saudi Arabia.

\section{Material and Method:-}

A search of published literatures that has addressed individual risk factors in Saudi Arabia was carried out via the internet, using the medical database MEDLINE/PubMed, supported by the US National Library of Medicine. The review was conducted to the time of December 2017. The methodology followed by the researcher for the process of selection of articles is presented in. The researchers conducted a review process and the discordances were resolved by consensus. At a first stage, all published articles containing the words "Prevalence", "Obesity or over weight", "Hypertension", "Diabetes", "Dyslipidemia", "Smoking", "Physical Inactivity" in combination with "Saudi Arabia" as part of their title, as a keyword or as a reference in their abstract have been collected. Initially, retrieved article were filtered confine the search results, excluding hospital based, case reports studies or none original papers. Finally, at the end selection process, we summarized our findings in this review article.

\section{Results:-}

Dyslipidemia:

Three landmark studies evaluated the prevalence of dyslipidemia in Saudi Arabia during the past 25 years. The first report came in 1996 by Al-Nuaim AR where he reported the outcome of community based cross sectional national epidemiological study involving almost 4500 Saudi citizens aging 15 years old or more. The investigators evaluated both the occurrence of hypercholesterolemia (HC) and mean serum total cholesterol (TC) in KSA. Collected data were: height, weight and random sample of blood for total cholesterol assays. The occurrence rate of HC (5.2-6.2 $\mathrm{mmol} / \mathrm{l})$ was $9 \%$ in men and $11 \%$ in women $(\mathrm{P}=0.74)$, whereas the occurrence rate of $\mathrm{HC}(>6.2 \mathrm{mmol} / \mathrm{l})$ was $7 \%$ among men and $8 \%$ in women $(\mathrm{P}=0.52)$. The prevalence of $\mathrm{HC}$ was conversely proportionate to diabetes and smoking as well. The mean serum TC in women was meaningfully higher than for men subjects (4.24 versus 4 mmol/l). A noticeable continuous rise in TC with age, attaining its peak at the 5th and 6th decades in all subjects. Also, there was positive correlation between TCC and increasing BMI values. [4].

In older age group 30-70 years of 16,819 Saudi subjects, Al-Nozha MM, assessed the prevalence of both HC, TC > $5.2 \mathrm{mmol} / \mathrm{l}$, and hypertriglyceridemia (HT), serum triglycerides $>1.69 \mathrm{mmol} / \mathrm{l}$, over 5 years period from 1996-2000. $\mathrm{HC}$ occurred in $54 \%$ and reported mean cholesterol level was 5.4+/-1.52 mmol/1. There was no significant variation in the prevalence of $\mathrm{HC}$ when gender or place of residence were considered. Hypertriglyceridemia occurrence was $40.3 \%$ and reported mean triglycerides level was 1.8+/-1.29 mmol/l. Men had statistically significant higher HT prevalence of $47.6 \%$ compared to $33.7 \%$ in females $(\mathrm{p}<0.0001)$. He concluded that dyslipidemia is reaching higher prevalence rates in Saudi Arabia and was able to predict that ischemic heart disease will evolve as major health issue soon. [5]

More recently in 2014, Basulaiman M, assessed the prevalence of HC in 2013 spring among 10,735 Saudi subjects who answered a specifically designed survey via face to face interviews and their age was 15 years and above. The overall result, $8.5 \%$ of citizens had $\mathrm{HC}$ and additional $19.6 \%$ were borderline hypercholesteremic. Among hypercholesterolemic Subjects, $65.1 \%$ were undiagnosed, $4.3 \%$ were untreated, $2.3 \%$ were uncontrolled despite being treated. Only $28.3 \%$ were controlled by lipid lowering therapy. The risk of HC increased in correlation to aging, margarine consumption, increased BMI and presence of comorbidities like diabetes or hypertension. [6] 


\section{Hypertension:}

In 1997, Al-Nozha MM conducted a cross-sectional community-based survey to investigate prevalence rate of hypertension in 13,700 Saudi subjects regardless of age or gender. Applying the WHO criteria for hypertension, 160/95 $\mathrm{mmHg}$, systolic hypertension prevalence was $9.1 \%$ and diastolic hypertension prevalence was $8.7 \%$. Hypertensive patients older than 40years old had $87.5 \%$ systolic and $79.4 \%$ diastolic hypertension. However, applying more stringent diagnostic cutoff for hypertension, 140/90 mmHg, subjects more than 40 years old were suffering from either systolic hypertension $20.4 \%$ or $25.9 \%$ diastolic hypertension. [7]

Ten years later, the same author investigated hypertension prevalence in a community- based study involving 17,230 subjects aging 30-70 years old. The study was conducted over 5 years period from 1995-2000. The crude prevalence of hypertension was $26.1 \%$. Men had significantly higher prevalence of hypertension compared to women $(28.6 \%$ vs. $23.9 \% \mathrm{p}<0.001)$ respectively. [8]

Al-Turki KA 2008, reported the outcome of a large screening campaign involving, conducted in the eastern province to evaluate hypertension prevalence during 2004 involving 197,681 subjects. JNC VII criteria for diagnosis of hypertension (140/90 mmHg) was applied to study samples and initially $21 \%$ of the subjects were positive for previous history or screening. After the next day confirmation, the prevalence of hypertension dropped to $15.6 \%$ and pre-hypertension accounted for 3.7\%. The investigators were able to highlight some differences regarding subjects' gender being significantly more prevalent in women than men, despite that, men had significantly higher mean systolic and diastolic blood pressure. [9]

A national health survey was conducted in 2013 involving 10,735 citizens older than 15 years. El Bacheraoui 2014, found that $15.2 \%$ were hypertensives and $40.6 \%$ were pre-hypertensives. Alarmingly, the diagnosis rate was 42.2 among hypertensive subjects. Among those diagnosed, almost $80 \%$ were treated and $45 \%$ of them achieved target blood pressure goals. [10]

\section{Diabetes Mellitus}

The earliest report regarding prevalence of diabetes in Saudi Arabia was reported by Fatani HH, 30 years ago. He and his colleagues screened 5222 subjects living in rural areas in western region. The prevalence rate of diabetes mellitus was $4.3 \%$. Prevalence of diabetes was higher among obese, higher income group and older population. Prevalence in females was double that for males. [11]

3 years later, Anokute CC, screened 3158 subjects from King Saud University Community for diabetes mellitus. The prevalence rate observed for fasting blood glucose $>140 \mathrm{mg} / \mathrm{dl}$ was $6.0 \%$. This study involved males only and diagnostic cutoff matched reference value at that period. [12]

In 1996, El-Hazmi, conducted a study to assess the prevalence of diabetes mellitus (DM) in variable areas of Saudi Arabia. A community survey that involved 23,493 Saudi females and males aging 2-70 years. Based on WHO diabetes diagnostic criteria, the overall prevalence of DM was 5.7\% men and $4.8 \%$ in women. In the 14-70-year age group, the prevalence of DM was $9.7 \%$ in the men and $7.1 \%$ and in women. In subjects $>30$ years, prevalence of T2 DM rose to $17.32 \%$ men and $12.18 \%$ in women. [13]

Al-Nuaim AR, in 1997 investigated prevalence of diabetes mellitus in Saudi citizens. 13,177 participants older than 15 years geographically distributed all over Saudi Arabia. Prevalence of DM, age adjusted, was among the highest in the world. Urban subjects had significantly higher prevalence (men 12\%, 95\% CI 11-13 and women $14 \%, 95 \%$ CI 13-15) than rural subjects (men 7\%, 95\% CI 7-8 and women 7.7\%, 95\% CI 7-9). [14]

In 2004, Al-Nuzha MM, published the outcome of population-based nationwide epidemiological survey, conducted by assessing Saudi subjects aging 30-70-years over a 5-year period, 1995 to 2000. Utilizing criteria of 1997 American Diabetes Association (ADA) that was endorsed by the WHO in 1998, the prevalence of DM was $23.7 \%$ among 16,917 participants. Men had a significantly higher prevalence than women $(26.2 \%$ vs. 21.5\% (p<0.00001) respectively. [15]

Further to this, in 2010 Al-Balghli NA, reported the outcome of DM screening campaign that took place in the eastern region during 2004 in subjects >30 years old. The confirmed prevalence rate of DM among study population 197,681 participants was $17.2 \%$. [16] 
El Bacheraoui 2014, analyzed data of the Saudi Health Interview Survey (SHIS) for diabetes mellitus prevalence estimation and concluded that diabetics represents $13.4 \%$ among Saudis $>15$ years old. Among those, 57.8\% are undiagnosed, $5.4 \%$ are untreated, $20.2 \%$ uncontrolled despite treatment and only $16.6 \%$ are achieving their treatment goals. [17]

Obesity and overweight

The following table summarizes all epidemiological studies conducted in Saudi Arabia (Table 1)

Table 1:- Prevalence of obesity (BMI >30 Kg/m2) in Saudi Arabia

\begin{tabular}{|c|c|c|c|c|c|c|c|c|}
\hline \multirow[b]{2}{*}{ Study (ref.) } & \multirow{2}{*}{ Data collection period } & \multicolumn{3}{|c|}{ Sample size (No.) } & \multirow{2}{*}{$\begin{array}{c}\text { Age groups } \\
\text { studied (years) }\end{array}$} & \multicolumn{3}{|c|}{ Obesity prevalence (\%) } \\
\hline & & Men & Women & Total & & Men & Women & Total \\
\hline Al-Nuaim et al. (18) & 1990-1993 & 5407 & 5244 & 10651 & $(\geq 20)$ & 17.8 & 26.6 & 22.1 \\
\hline Osman \& Al-Nozha (19) & 1989-1994 & 2673 & 3590 & 6263 & $(\geq 18)$ & 15.6 & 24.9 & 20.8 \\
\hline Warsy \& El-Hazmi (20) & 1992-1995 & 6646 & 9064 & 15710 & $(>14)$ & 13.1 & 20.3 & 15.8 \\
\hline Al-Nuzha et al (21) & $1995-2000$ & & & 17232 & $(30-70)$ & 26.4 & 44 & 35.6 \\
\hline WHO STEP wise (22) & 2005 & 2244 & 2345 & 4589 & $(15-64)$ & 28.3 & 43.8 & 36.2 \\
\hline Memish et al (23) & 2013 & & & 10735 & $(>15)$ & 24.1 & 33.5 & 28.7 \\
\hline
\end{tabular}

Obesity is a public health burden in Saudi Arabia impacting negatively not only the quality of life but also morbidity and even mortality outcomes.

\section{Physical Inactivity}

Al-Hazzaa HM 2004, reviewed the current literatures discussing physical inactivity in Saudi population. The reported prevalence of physical inactivity ranges from 43.3-99.5\% among children and even adults. [24]

\section{Smoking}

In 2015, Moradi-Lakeh, published results of a survey on 10735 subjects >15 years old (5253 males and 5482 females) that took place during spring 2013. Current overall smokers were $12.2 \%$. Men were more likely to smoke than women $(21.5 \%$ vs. $1.1 \%)$. [25]

\section{Conclusion:-}

ASCVD major modifiable risk factors including dyslipidemia, hypertension, diabetes mellitus, obesity, physical inactivity and smoking are quite prevalent among Saudi population and despite being captured in literatures for almost 30 years still their trend is growing. There is a need for more comprehensive awareness programs to publics aiming to promote healthy lifestyle and enhance early detection and adherence to treatment.

\section{Financial Disclosure:-}

Self-funded

\section{Authors Contribution:-}

All authors contributed to this article including, searching, filtering, manuscript writing, reviewing and editing.

\section{References:-}

1. Ali MK, Bullard KM, Saaddine JB, Cowie CC, Imperatore G, Gregg EW. Achievement of goals in U.S. diabetes care, 1999-2010. N Engl J Med 2013;368:1613-1624

2. World Heart Day in WHO Cardiovascular Diseases accessed on 20 Jan. 2018 at http://www.who.int/cardiovascular_diseases/world-heart-day-2017/en/

3. World Heart Day in WHO Cardiovascular Diseases accessed on 20 Jan. 2018 at http://www.who.int/cardiovascular_diseases/priorities/en/

4. Al-Nuaim AR et al. Prevalence of hypercholesterolemia in Saudi Arabia, epidemiological study. Int J Cardiol. 1996 Apr 19;54(1):41-9.

5. Al-Nozha MM et al. Hyperlipidemia in Saudi Arabia. Saudi Med J. 2008 Feb;29(2):282-7.

6. Basulaiman $\mathrm{M}$ et al. Hypercholesterolemia and its associated risk factors-Kingdom of Saudi Arabia, 2013. Ann Epidemiol. 2014 Nov;24(11):801-8.

7. Al-Nozha MM et al. Arterial hypertension in Saudi Arabia. Ann Saudi Med. 1997 Mar;17(2):170-4.

8. Al-Nozha MM et al. Hypertension in Saudi Arabia.Saudi Med J. 2007 Jan;28(1):77-84. 
9. Al-Turki KA et al. Hypertension in the eastern province of saudi arabia: results of a screening campaign. $\mathrm{J}$ Family Community Med. 2008 Sep;15(3):95-101.

10. El Bcheraoui $\mathrm{C}$ et al. Hypertension and its associated risk factors in the kingdom of saudi arabia, 2013: a national survey. Int J Hypertens. 2014;2014:564-679.

11. Fatani HH et al. Prevalence of diabetes mellitus in rural Saudi Arabia. Diabetes Care. 1987 Mar-Apr;10(2):1803.

12. Anokute $\mathrm{CC}$ et al. Epidemiologic studies of diabetes mellitus in Saudi Arabia--Part I--Screening of 3158 males in King Saud University. J R Soc Health. 1990 Dec;110(6):201-3.

13. El-Hazmi MA et al. Diabetes mellitus and impaired glucose tolerance in Saudi Arabia.Ann Saudi Med. 1996 Jul;16(4):381-5.

14. Al-Nuaim AR et al. Prevalence of glucose intolerance in urban and rural communities in Saudi Arabia. Diabet Med. 1997 Jul;14(7):595-602.

15. Al-Nozha MM et al. Diabetes mellitus in Saudi Arabia. Saudi Med J. 2004 Nov;25(11):1603-10.

16. Al-Baghli NA et al. Prevalence of diabetes mellitus and impaired fasting glucose levels in the Eastern Province of Saudi Arabia: results of a screening campaign. Singapore Med J. 2010;51(12):923-30.

17. El Bcheraoui C et al. Status of the diabetes epidemic in the Kingdom of Saudi Arabia, 2013. Int J Public Health. 2014 Dec;59(6):1011-21.

18. Al-Nuaim AA et al. Overweight and obesity in Saudi Arabian adult population, role of socio-demographic variables. J Community Health1997;22(3):211-23.

19. Osman AK, Al-Nozha MM. Risk factors of coronary artery disease in different regions of Saudi Arabia. East Mediterr Health J. 2000 Mar-May;6(2-3):465-74.

20. Warsy AS, El-Hazmi MA. Diabetes mellitus, hypertension and obesity-common multifactorial disorders in Saudis. East Mediterr Health J. 1999 Nov;5(6):1236-42.

21. Al-Nozha MM et al. Obesity in Saudi Arabia. Saudi Med J. 2005 May;26(5):824-9.

22. WHO STEP wise approach to NCD surveillance. Country specific standard report, Saudi Arabia 2004. Riyadh: Ministry of Health, Saudi Arabia, and World Health Organization; 2005 www.who.int/chp/steps/2005_SaudiArabia_STEPS_Report_EN.pdf, accessed 20 Jan 2018).

23. Memish ZA et al. Obesity and Associated Factors - Kingdom of Saudi Arabia, 2013. Prev Chronic Dis 2014;11:140236.

24. Al-Hazzaa HM. Prevalence of physical inactivity in Saudi Arabia: a brief review. East Mediterr Health J. 2004;10(4-5):663-70.

25. Moradi-Lakeh $M$ et al.Tobacco consumption in the Kingdom of Saudi Arabia, 2013: findings from a national survey. BMC Public Health. 2015; 15: 611. 